EISSN: 2706-7955 ISSN: 2077-4605

DOI: 10.36632/mejar/2021.10.1.5

Journal homepage: www.curresweb.com

Pages: 73-91

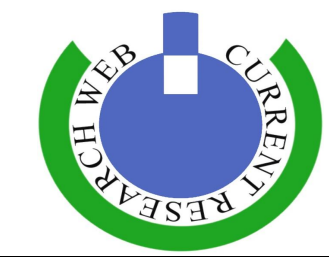

\title{
Study on Genesis and Parent Material Homogeneity of Some Soils West Manfalut, Assuit, Egypt
}

\author{
Aref A. A. ${ }^{1}$, El Kady M. M. ${ }^{2}$, Faragallah M. A. ${ }^{1}$, El-Desoky A. I. $^{1}$ \\ ${ }^{1}$ Department of Soils and Water, Faculty of Agriculture, Al-Azhar University, Assiut, Egypt \\ ${ }^{2}$ Pedology Department, Desert Research Centre (DRC), El-Matariya 11753, Cairo, Egypt
}

Received: 15 December 2020 Accepted: 15 January $2021 \quad$ Published: 25 January 2021

\begin{abstract}
The main objective of this paper is to appraise genesis and parent material uniformity along a toposequence of some soils west Manfalut, Assuit-Egypt for precision of agricultural landuse management. A total of forty soil samples were collected from sixteen soil profiles that represent most of the ground elevations of the study area ( 35 to $125 \mathrm{~m}$ a.s.l). These samples were used for heavy minerals identification. The soils were deep having a texture between sand and sandy clay loam with varied contents of gravel. The highest frequency of Opaques and Ubiquitous was found in the north of the research area (86.9 m a.s.1). Further, Pyroboles and Para-metamorphic minerals were detected in maximum contents in the south portion (59.4 \& $111.1 \mathrm{~m}$ a.s.1), respectively. A significant positively correlation was found between Augite \& Apatite, while a significant negatively one was observed between Tourmaline \& Zircon. Maturity index values were dominantly less than $75 \%$, indicating weakly developed soils. Morphological characterization and vertical distribution of $\mathrm{Z} / \mathrm{T}, \mathrm{Z} / \mathrm{R}$, and $\mathrm{Z} / \mathrm{R}+\mathrm{T}$ showed that parent materials of the investigated soils are apparently formed of heterogeneous depositional regimes. Accordingly, these soils have particular landuse restrictions and require moderate to special conservation practices for cultivation and providing permanent protection.
\end{abstract}

Keywords: Soil genesis; Parent material, Heavy minerals, Manfalut, Arid desert, Landuse

\section{Introduction}

Advanced investigations of land resources for sustainable use along with the implementation of effective land-use management techniques need to realize of pedogenesis and parent material homogeneity (El Kady and Yossif 2017).

Previous studies indicate that uniformity of the parent material is a requirement for assessing pedological processes. Minerals that are immune to chemical weathering and immobile in soils have been a powerful basis for evaluating horizon variations within pedons. In order to distinguish between geological and pedological processes, the distribution of resistant minerals in the solum was used. (Barshad 1965, Brewer 1976, Taboada et al., 2006).

Moreover, characterization of lithological discontinuities which have considerable changes in mineralogical composition is decisive to pedogenesis interpretation and appraisement (Chapman and Horn 1968, Raad and Prozt 1971, Schaetzl, 1998).

Zircon $(Z)$, rutile $(R)$, tourmaline $(T)$ and quartz are resistant minerals and they were appointed for evaluating pedogenesis and parent material uniformity. The vertical homogeneity in their frequency distribution within the soil profile is known as evidence that the parent material was uniform. If their content $(\mathrm{Z}+\mathrm{R}+\mathrm{T})$ is more than 75 percent, the inference of pedological processes may either be or have been continued (Hubert 1962).

In many pedogenesis researches, discrimination of soil parent material uniformity is important and valuable since it determines whether observed differences in morphological, physiochemical and mineralogical characteristics are the conclusion of genetic processes or inherited material divergences 
(Norton and Hall 1984). Wilson et al., (2017) mentioned that pedogenesis appraisement and its related factors are important for soil survey, agronomy/soil fertility and land-use management.

Newly reclaimed soils in hyper-arid regions like that located in western Manfalut, Assuit-Egypt, are prone frequently to a net addition of ions over time (Gile et al., 1981). This anthropogenic impact can lead to a variation in distinctive pedogenic mineral accumulations and soil horizons, hence might affect the situation of pedogenesis and parent materials discontinuity with depth.

\subsection{An understanding of the interactions}

Therefore, perception of soil genesis and evaluation of parent materials uniformity in western Manfalut, Assuit-Egypt is required to foretell the nature and occurrence of soils and how they will respond to assorted land managements. So, the paper aims to assess genesis and parent material homogeneity along a topo-sequence of some soils west Manfalut, Assuit-Egypt for precision of agricultural landuse management.

\section{Materials and Methods}

\subsection{Site description}

The area under investigation is located in the western portion of Manfalut, Assuit-Egypt that represents a new reclaimed and promised site for agriculture. It stretches between longitudes $30^{\circ} 50^{\prime} 10^{\prime \prime}$ and $30^{\circ} 57^{\prime} 55^{\prime \prime} \mathrm{E}$, and latitudes $27^{\circ} 13^{\prime} 41^{\prime \prime}$ and $27^{\circ} 18^{\prime} 27.5^{\prime \prime} \mathrm{N}$, occupying an area of about $76 \mathrm{~km}^{2}$. Additionally, it has a variation of surface elevations ranges between 35-125 m a.s.l (Fig. 1).

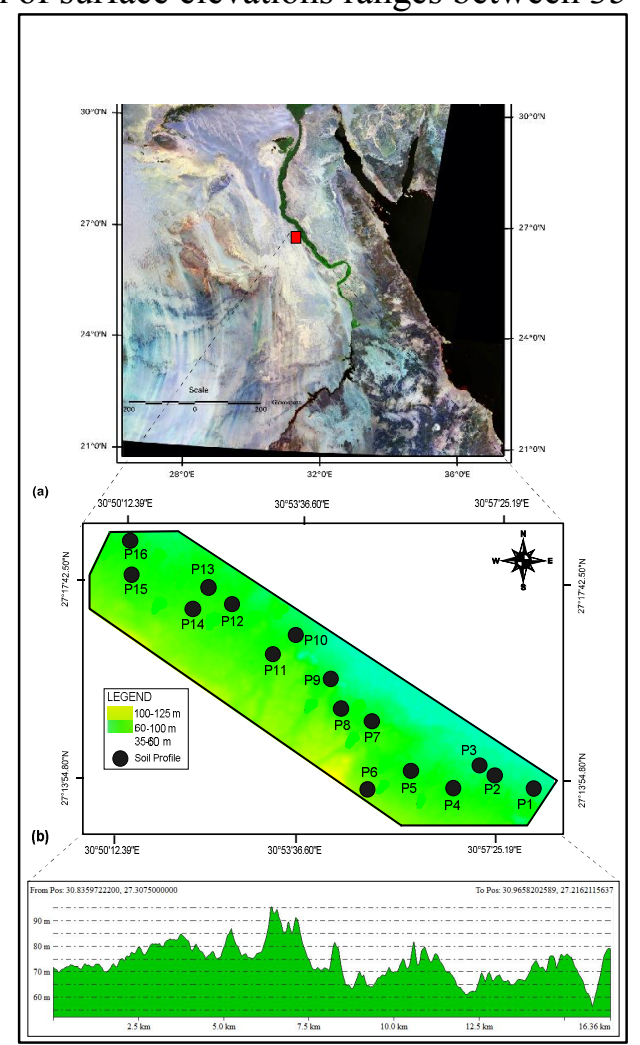

Fig. 1: (a) Location of the study area, (b) the topo-sequence model and geographic locations of the selected soil profiles

Geologically, the study area is essentially occupied by sediments belonging to the Quaternary and Tertiary formations, (Fig. 2). Quaternary sediments represented in sand dunes, Nile deposits, and others (i.e. fanglomerate) while Tertiary formations were mainly Eocene (i.e. thick marine limestone with chert and minor clay beds), (Said 1962 and 1981, Omer 1996, Osman 1980, Geological Survey of Egypt 1981). 


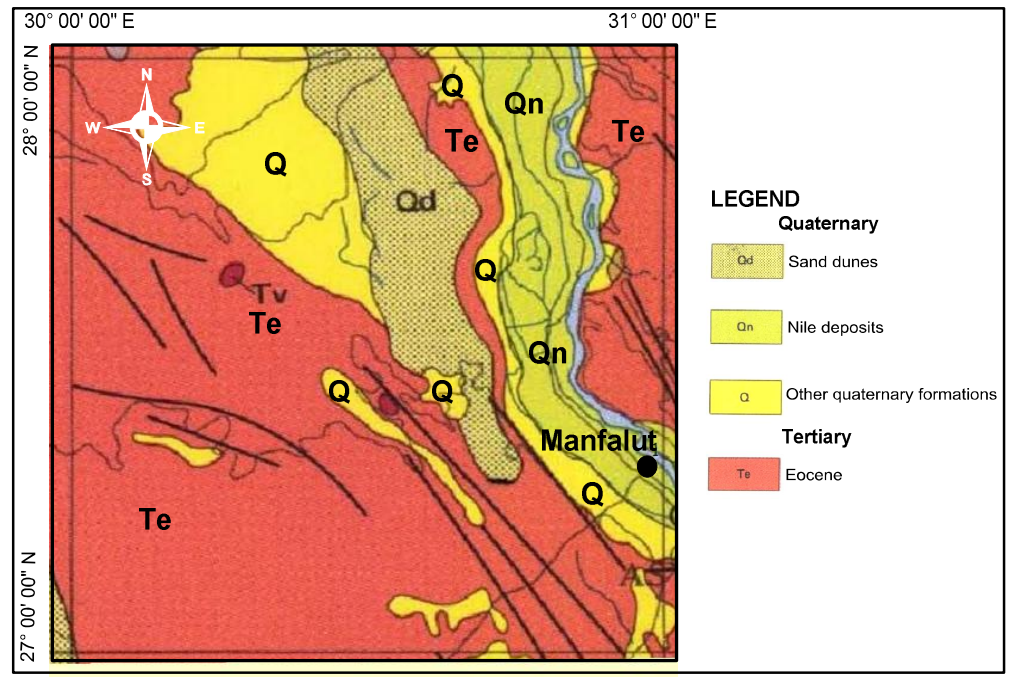

Fig. 2: Geological map of the study area (modified after Geological Survey of Egypt, 1981)

Three geomorphic units could be distinguished west Manfalut: (1) old alluvial plain (sand and gravel), (2) young alluvial plain (silty clay cultivated lands), and (3) calcareous structural plateau (Eocene limestone covered by drift sands, flints, and boulders of carbonate), (Abou El-Anwar et al., 2019), (Fig. 3). However, the study soils located within the first unite only.

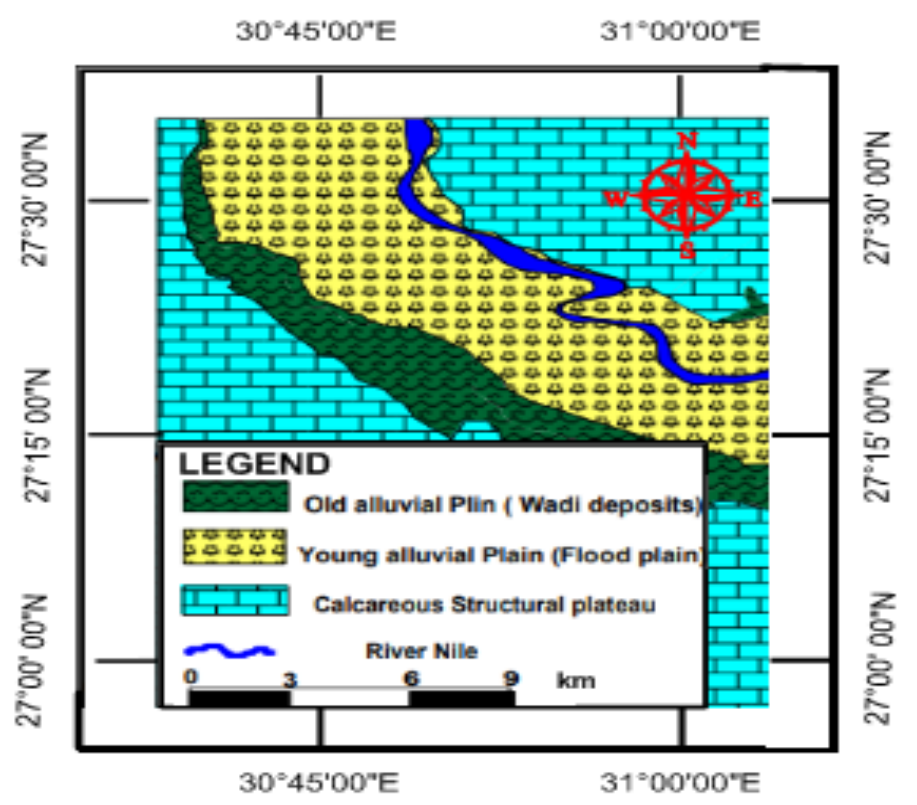

Fig. 3: Geomorphological map of west Manfalut, Assuit (modified after Abou El-Anwar et al., 2019)

\subsection{Soil sampling and analyses}

Sixteen soil profiles were selected to determine heavy minerals of the sand fraction (63-125 $\mu)$ in the area of investigation. Sites of soil profiles were chosen to give representative selection of the variability in elevations (35 to $125 \mathrm{~m}$ a.s.1) on basis of a topo-sequence transect from Northwest to Southeast direction with a length about of $16 \mathrm{Km}$ within the study area, (Fig. 1). The profiles till a depth of almost 110-150 cm were exposed and main macro-morphological features were described following the guidelines set by Soil Survey Manual (Soil Survey Staff 2009). The genetic horizons were described, and selected soil samples were collected from some of them (total soil samples $=40$ ) for mineralogical analysis (Table 1).

Soil samples were air-dried and coarse fragments $>2 \mathrm{~mm}$ were removed by sieving and the soil samples $(<2 \mathrm{~mm})$ were prepared for estimation of heavy minerals using methods in Brewer (1964). Hence, the sand fractions were treated with $0.1 \mathrm{~N} \mathrm{HCl}$ for carbonate removal, $30 \% \mathrm{H}_{2} \mathrm{O}_{2}$ for organic 
matter and manganese oxides removal and sodium dithionite for free iron oxides removal and sodium dithionite for free iron oxides removal. The sand fractions were washed with distilled water to ensure the removal of soluble salts, dried and the fraction of $(63-125 \mu)$ was separated by dry sieving for heavy minerals examination. Separation of heavy minerals was carried out using the bromoform separation technique.

Minerals were recognized and estimated by polarizing microscope. The principles applied for identification through optical properties of minerals are those given by (Jay 2015, Mallik 2018). Approximately 500 grains in each slide were examined to minimize the absolute error, (Mahaney 2002).

\subsection{Calculation of maturity index $\&$ uniformity ratios}

Zircon $(Z)$, rutile $(R)$ and tourmaline $(T)$ percentages were used to calculate maturity index of the investigated soils using the following equation: $Z T R=(Z+T+R / \text { Non-opaque minerals })^{*} 100$. Hubert (1962) schemed to this equation and referred that the values of ZTR less than $75 \%$ implies immature to sub-mature sediments; ZTR more than $75 \%$ indicates matured sediments from the mineralogical point of view. The ratios of $\mathrm{Z} / \mathrm{R}, \mathrm{Z} / \mathrm{T}, \mathrm{Z} / \mathrm{R}+\mathrm{T}$ were calculated as evidences for uniformity or heterogeneity of parent materials, according to (Haseman and Marshall 1945, Hammad 1968, El Kady 2008).

\subsection{Statistical analysis}

Correlation analyses for heavy minerals contents among each to others were determined using the SPSS software 16.0 following the procedures detailed by (Reid 2013).

\section{Results and Discussion}

\subsection{Soil Morphology}

The soils were deep as it ranged from $110-150 \mathrm{~cm}$ and exhibited variation in texture ranging between sand and sandy clay loam (Table 1). Soil colour matrix exhibited 10YR hue, 5 to 7 value, and 2 to 6 chroma in dry state, reflecting that soils are composed of different parent materials. Coarse fragments were observed within all the studied pedons and varied broadly with a volume not more than $64 \%$ of medium gravel. This could be attributed to the nature of soil parent materials forming the studied area. The subangular blocky structure was dominant at the upper horizons, while the lower ones shaped with either single grain or angular blocky. Surface soils characterized by soft consistence, while subsurface ones ranged from slightly hard to very hard or completely loose. Stickiness and plasticity of the investigated soils fluctuated between none-sticky, none-plastic and sticky, plastic consistency.

Roots were found at most of soil layers where it covered $\mathrm{C} 1$ horizons with a considerable abundance of medium size which it decreased with depth. The soils had slightly to violently effervescent reaction with $\mathrm{HCl}$, which is ascribed to the lithological composition of the studied site. Boundaries between soil horizons ranged between abrupt to clear, referring to apparent differences in the mode of deposition between the genetic horizons of the soils. Accordingly, most of these soils might be classified as Typic Torriorthents, (USDA 2014).

\subsection{Heavy mineral composition and distribution}

The quantitative mineral analysis of the very fine sand fraction $(0.125-0.063 \mathrm{~mm})$ of the soil samples and its statistical analysis are given in tables 2 and 3 for the heavy minerals. Heavy mineral assemblage of the study region is restricted to the dominance of Opaques, Augite, Hypersthene, Hornblende, Garnet, Sillimanite, and Apatite along with few other non-opaques. The opaque minerals $(\mathrm{OM})$ comprised mainly of Ilmenite, Magnetite, Hematite, and Goethite and found in considerable amounts in most of samples. The surface soil of profile (14) at ground elevation of $86.9 \mathrm{~m}$ a.s.l was the higher percentage rather than the others. Furthermore, no clear trends of variations in such minerals could be noted along the topo-sequence model of the studied area. The highest value of positive correlation was between OM and rutile $(r=0.19)$, while the maximum one of negative correlation was with Sillimanite $(r=-0.22)$.

Augite, Hypersthene, Hornblende were the prevailing members of pyroboles group within the investigated soils. Augite and Hornblende occupied all the estimated soil samples except Hypersthene which is mostly found in minor quantities or not observed. Vertical distribution of total pyroboles 
Table 1: Morphological characteristics of the investigated soils along with the ground elevation variations

\begin{tabular}{|c|c|c|c|c|c|c|c|c|c|c|c|}
\hline \multirow{2}{*}{ Horizon } & \multirow{2}{*}{$\begin{array}{c}\text { Depth } \\
\text { (cm) }\end{array}$} & \multicolumn{2}{|c|}{ Colour } & \multirow{2}{*}{$\begin{array}{c}\text { Coarse } \\
\text { fragments } \\
\text { Vol }(\%)^{1}\end{array}$} & \multirow{2}{*}{$\begin{array}{l}\text { Texture } \\
\text { USDA }^{2}\end{array}$} & \multirow{2}{*}{ Structure $^{3}$} & \multicolumn{2}{|c|}{ Consistence $^{4}$} & \multirow{2}{*}{ Roots $^{5}$} & \multirow{2}{*}{$\begin{array}{c}\text { Reaction } \\
\text { with } \mathbf{H C l}^{6}\end{array}$} & \multirow{2}{*}{ Boundary $^{7}$} \\
\hline & & dry & moist & & & & dry & wet & & & \\
\hline \multicolumn{12}{|c|}{ Profile No.1, (63.4 m a.s.l) } \\
\hline $\mathrm{C} 1$ & $0-15$ & $10 \mathrm{YR} 7 / 4$ & 10 YR 5/4 & $\mathrm{cmg}$ & $\mathrm{sl}$ & sbk & $\mathrm{s}$ & $\mathrm{ss}, \mathrm{ps}$ & $\mathrm{mm}$ & es & as \\
\hline $\mathrm{C} 2$ & $15-45$ & $10 \mathrm{YR} 7 / 3$ & 10 YR 5/4 & $\mathrm{cmg}$ & $\mathrm{s}$ & sg & 1 & so, po & $\mathrm{mc}$ & es & aw \\
\hline $2 \mathrm{C} 3$ & $45-75$ & $10 \mathrm{YR} 7 / 3$ & 10 YR 5/4 & fmg & $\mathrm{s}$ & $\mathrm{sg}$ & 1 & so, po & $\mathrm{ff}$ & es & cs \\
\hline $\mathrm{C} 4$ & $75-150$ & $10 \mathrm{YR} 7 / 3$ & 10 YR 5/4 & fmg & ls & $\mathrm{m}$ & $\mathrm{h}$ & ss, po & - & es & - \\
\hline \multicolumn{12}{|c|}{ Profile No.2, (61.9 $\mathrm{m}$ a.s.l) } \\
\hline $\mathrm{C} 1$ & $0-10$ & $10 \mathrm{YR} 7 / 3$ & $10 \mathrm{YR} 5 / 4$ & $\mathrm{cmg}$ & $\mathrm{s}$ & $\mathrm{m}$ & $\mathrm{s}$ & so, po & $\mathrm{mm}$ & e & as \\
\hline $\mathrm{C} 2$ & $10-20$ & $10 \mathrm{YR} 7 / 3$ & 10 YR 5/4 & $\mathrm{cmg}$ & ls & $\mathrm{sg}$ & 1 & ss, po & $\mathrm{mm}$ & es & ai \\
\hline $\mathrm{C} 3$ & $20-40$ & $10 \mathrm{YR} 7 / 3$ & 10 YR 5/4 & $\mathrm{cmg}$ & $\mathrm{s}$ & $\mathrm{sg}$ & 1 & so, po & $\mathrm{mm}$ & es & cs \\
\hline $2 \mathrm{C} 4$ & $40-65$ & $10 \mathrm{YR} 7 / 4$ & 10 YR 5/6 & fmg & $\mathrm{s}$ & $\mathrm{m}$ & $\mathrm{h}$ & so, po & $\mathrm{ff}$ & $\mathrm{e}$ & - \\
\hline $\mathrm{C} 5$ & $65-95$ & $10 \mathrm{YR} 7 / 4$ & 10 YR 5/6 & $\mathrm{cmg}$ & ls & $\mathrm{m}$ & sh & ss, po & - & e & cs \\
\hline C6 & $95-150$ & $10 \mathrm{YR} 7 / 3$ & 10 YR 5/6 & $\mathrm{cmg}$ & $\mathrm{sl}$ & abk & $\mathrm{h}$ & ss, ps & - & e & - \\
\hline \multicolumn{12}{|c|}{ Profile No.3, (59.4 m a.s.l) } \\
\hline $\mathrm{C} 1$ & $0-15$ & $10 \mathrm{YR} 7 / 3$ & $10 \mathrm{YR} 5 / 3$ & $\mathrm{cmg}$ & sl & sbk & $\mathrm{s}$ & ss, ps & - & es & as \\
\hline $\mathrm{C} 2$ & $15-35$ & 10 YR 7/4 & 10 YR 5/4 & $\mathrm{cmg}$ & $\mathrm{s}$ & $\mathrm{sg}$ & 1 & so, po & - & e & aw \\
\hline $\mathrm{C} 3$ & $35-110$ & $10 \mathrm{YR} 7 / 4$ & 10 YR 5/6 & $\mathrm{mmg}$ & sl & abk & $\mathrm{h}$ & ss, ps & - & es & - \\
\hline
\end{tabular}


Table 1: Cont.

\begin{tabular}{|c|c|c|c|c|c|c|c|c|c|c|c|}
\hline \multirow{2}{*}{ Horizon } & \multirow{2}{*}{$\begin{array}{c}\text { Depth } \\
\text { (cm) }\end{array}$} & \multicolumn{2}{|c|}{ Colour } & \multirow{2}{*}{$\begin{array}{c}\text { Coarse } \\
\text { fragments } \\
\operatorname{Vol}(\%)^{1}\end{array}$} & \multirow{2}{*}{$\begin{array}{l}\text { Texture } \\
\text { USDA }^{2}\end{array}$} & \multirow{2}{*}{ Structure $^{3}$} & \multicolumn{2}{|c|}{ Consistence $^{4}$} & \multirow{2}{*}{ Roots $^{5}$} & \multirow{2}{*}{$\begin{array}{c}\text { Reaction } \\
\text { with } \mathrm{HCl}^{6}\end{array}$} & \multirow{2}{*}{ Boundary $^{7}$} \\
\hline & & Dry & Moist & & & & Dry & Wet & & & \\
\hline \multicolumn{12}{|c|}{ Profile No.4, (76.4 m a.s.l) } \\
\hline $\mathrm{C} 1$ & $0-15$ & $10 \mathrm{YR} 7 / 6$ & 10 YR 5/6 & amg & ls & $\mathrm{m}$ & $\mathrm{s}$ & ss, po & $\mathrm{mm}$ & es & as \\
\hline $2 \mathrm{C} 2$ & $15-55$ & $10 \mathrm{YR} 7 / 4$ & 10 YR 5/6 & $\mathrm{mmg}$ & ls & $\operatorname{sg}$ & 1 & ss, po & $\mathrm{mm}$ & es & aw \\
\hline $\mathrm{C} 3$ & $55-80$ & $10 \mathrm{YR} 7 / 6$ & 10 YR 5/6 & $\mathrm{mmg}$ & sl & $\mathrm{m}$ & $\mathrm{s}$ & ss, ps & $\mathrm{ff}$ & es & cs \\
\hline $\mathrm{C} 4$ & $80-130$ & $10 \mathrm{YR} 5 / 4$ & $10 \mathrm{YR} 4 / 4$ & $\mathrm{cmg}$ & sil & abk & $\mathrm{h}$ & ss, ps & - & es & as \\
\hline $\mathrm{C} 5$ & $130-150$ & $10 \mathrm{YR} 5 / 3$ & $10 \mathrm{YR} 4 / 3$ & $\mathrm{mmg}$ & $\mathrm{scl}$ & abk & v.h & $s, p$ & - & $\mathrm{e}$ & - \\
\hline \multicolumn{12}{|c|}{ Profile No.5, (74.3 m a.s.l) } \\
\hline $\mathrm{C} 1$ & $0-10$ & $10 \mathrm{YR} 7 / 3$ & 10 YR 5/6 & $\mathrm{cmg}$ & sl & sbk & $\mathrm{s}$ & ss, ps & $\mathrm{mm}$ & es & as \\
\hline $\mathrm{C} 2$ & $10-30$ & $10 \mathrm{YR} 7 / 4$ & 10 YR 5/6 & $\mathrm{cmg}$ & ls & sg & 1 & ss, po & $\mathrm{mm}$ & es & aw \\
\hline $\mathrm{C} 3$ & $30-70$ & 10 YR 7/4 & 10 YR 5/6 & $\mathrm{cmg}$ & $\mathrm{s}$ & sg & 1 & so, po & $\mathrm{ff}$ & es & cs \\
\hline $\mathrm{C} 4$ & $70-140$ & 10 YR 7/6 & 10 YR 5/6 & $\mathrm{mmg}$ & ls & $\mathrm{m}$ & $\mathrm{h}$ & ss, po & - & es & - \\
\hline \multicolumn{12}{|c|}{ Profile No.6, (111.1 m a.s.l) } \\
\hline $\mathrm{C} 1$ & $0-15$ & $10 \mathrm{YR} 7 / 3$ & 10 YR 5/6 & vfmg & sl & sbk & $\mathrm{s}$ & ss, ps & $\mathrm{mm}$ & es & as \\
\hline $\mathrm{C} 2$ & $15-95$ & 10 YR 7/6 & 10 YR 5/6 & $\mathrm{mmg}$ & ls & $\mathrm{sg}$ & 1 & ss, po & $\mathrm{ff}$ & ev & aw \\
\hline $2 \mathrm{C} 3$ & $95-115$ & 10 YR 5/4 & 10 YR 4/4 & $\mathrm{mmg}$ & $\mathrm{s}$ & sg & 1 & so, po & - & es & aw \\
\hline $2 \mathrm{C} 4$ & $115-150$ & 10 YR 7/4 & 10 YR 6/4 & vfmg & $\mathrm{s}$ & $\mathrm{sg}$ & 1 & so, po & - & es & - \\
\hline
\end{tabular}


Table 1: Cont.

\begin{tabular}{|c|c|c|c|c|c|c|c|c|c|c|c|}
\hline \multirow{2}{*}{ Horizon } & \multirow{2}{*}{$\begin{array}{c}\text { Depth } \\
\text { (cm) }\end{array}$} & \multicolumn{2}{|c|}{ Colour } & \multirow{2}{*}{$\begin{array}{c}\text { Coarse } \\
\text { fragments } \\
\text { Vol }(\%)^{1}\end{array}$} & \multirow{2}{*}{$\begin{array}{l}\text { Texture } \\
\text { USDA }^{2}\end{array}$} & \multirow{2}{*}{ Structure $^{3}$} & \multicolumn{2}{|c|}{ Consistence $^{4}$} & \multirow{2}{*}{$\operatorname{Roots}^{5}$} & \multirow{2}{*}{$\begin{array}{c}\text { Reaction } \\
\text { with } \mathrm{HCl}^{6}\end{array}$} & \multirow{2}{*}{ Boundary $^{7}$} \\
\hline & & Dry & Moist & & & & dry & wet & & & \\
\hline \multicolumn{12}{|c|}{ Profile No.7, (82.3 m a.s.l) } \\
\hline $\mathrm{C} 1$ & $0-30$ & $10 \mathrm{YR} 7 / 6$ & $10 \mathrm{YR} 5 / 6$ & $\mathrm{cmg}$ & $\mathrm{s}$ & $\mathrm{sg}$ & 1 & so, po & $\mathrm{mm}$ & es & as \\
\hline $\mathrm{C} 2$ & $30-85$ & $10 \mathrm{YR} 7 / 3$ & $10 \mathrm{YR} 5 / 4$ & amg & ls & sg & 1 & ss, po & $\mathrm{mm}$ & e & aw \\
\hline $\mathrm{C} 3$ & $85-140$ & $10 \mathrm{YR} 7 / 4$ & 10 YR 5/6 & $\mathrm{cmg}$ & $\mathrm{s}$ & $\mathrm{sg}$ & 1 & so, po & - & e & - \\
\hline \multicolumn{12}{|c|}{ Profile No.8, (87.5 m a.s.l) } \\
\hline $\mathrm{C} 1$ & $0-15$ & $10 \mathrm{YR} 6 / 2$ & $10 \mathrm{YR} 4 / 2$ & $\mathrm{cmg}$ & $\mathrm{scl}$ & sbk & s.h & $\mathrm{s}, \mathrm{p}$ & $\mathrm{mm}$ & es & as \\
\hline $\mathrm{C} 2$ & $15-55$ & $10 \mathrm{YR} 7 / 3$ & $10 \mathrm{YR} 5 / 4$ & $\mathrm{cmg}$ & 1 & sbk & $\mathrm{h}$ & ss, ps & $\mathrm{ff}$ & es & aw \\
\hline $\mathrm{C} 3$ & $55-110$ & $10 \mathrm{YR} 7 / 3$ & 10 YR 5/4 & fmg & ls & sg & 1 & ss, po & - & es & - \\
\hline \multicolumn{12}{|c|}{ Profile No.9, (71.8 $\mathrm{m}$ a.s.l) } \\
\hline $\mathrm{C} 1$ & $0-15$ & $10 \mathrm{YR} 7 / 3$ & 10 YR $5 / 3$ & $\mathrm{cmg}$ & ls & $\mathrm{m}$ & $\mathrm{s}$ & ss, po & $\mathrm{mm}$ & es & aw \\
\hline $2 \mathrm{C} 2$ & $15-35$ & $10 \mathrm{YR} 7 / 3$ & 10 YR $5 / 4$ & $\mathrm{cmg}$ & ls & sg & 1 & ss, po & $\mathrm{mm}$ & es & as \\
\hline $2 \mathrm{C} 3$ & $35-70$ & $10 \mathrm{YR} 7 / 3$ & 10 YR 5/4 & fmg & ls & $\mathrm{sg}$ & 1 & ss, po & $\mathrm{ff}$ & es & aw \\
\hline $\mathrm{C} 4$ & $70-150$ & $10 \mathrm{YR} 7 / 3$ & 10 YR 5/4 & $\mathrm{cmg}$ & sl & sbk & $\mathrm{h}$ & ss, ps & - & es & - \\
\hline
\end{tabular}


Table 1: Cont.

\begin{tabular}{|c|c|c|c|c|c|c|c|c|c|c|c|}
\hline \multirow{2}{*}{ Horizon } & \multirow{2}{*}{$\begin{array}{c}\text { Depth } \\
\text { (cm) }\end{array}$} & \multicolumn{2}{|c|}{ Colour } & \multirow{2}{*}{$\begin{array}{c}\text { Coarse } \\
\text { fragments } \\
\text { Vol }(\%)^{1}\end{array}$} & \multirow{2}{*}{$\begin{array}{l}\text { Texture } \\
\text { USDA }^{2}\end{array}$} & \multirow{2}{*}{ Structure $^{3}$} & \multicolumn{2}{|c|}{ Consistence $^{4}$} & \multirow{2}{*}{ Roots $^{5}$} & \multirow{2}{*}{$\begin{array}{l}\text { Reaction with } \\
\text { HCI }^{6}\end{array}$} & \multirow{2}{*}{ Boundary } \\
\hline & & Dry & Moist & & & & Dry & Wet & & & \\
\hline \multicolumn{12}{|c|}{ Profile No.10, (71.6 m a.s.l) } \\
\hline $\mathrm{C} 1$ & $0-20$ & $10 \mathrm{YR} 7 / 3$ & $10 \mathrm{YR} 5 / 3$ & $\mathrm{cmg}$ & sl & sbk & $\mathrm{s}$ & ss, ps & $\mathrm{mm}$ & es & as \\
\hline $2 \mathrm{C} 2$ & $20-60$ & $10 \mathrm{YR} 7 / 3$ & $10 \mathrm{YR} 5 / 4$ & fmg & sl & abk & s.h & ss, ps & $\mathrm{mm}$ & $\mathrm{e}$ & aw \\
\hline $\mathrm{C} 3$ & $60-120$ & $10 \mathrm{YR} 7 / 3$ & $10 \mathrm{YR} 5 / 4$ & $\mathrm{cmg}$ & ls & $\mathrm{sg}$ & 1 & ss, po & - & es & - \\
\hline \multicolumn{12}{|c|}{ Profile No.11, (83.4 m a.s.l) } \\
\hline $\mathrm{C} 1$ & $0-15$ & $10 \mathrm{YR} 7 / 4$ & $10 \mathrm{YR} 5 / 4$ & $\mathrm{cmg}$ & $\mathrm{sl}$ & sbk & $\mathrm{s}$ & ss, ps & $\mathrm{mm}$ & es & as \\
\hline $2 \mathrm{C} 2$ & $15-35$ & $10 \mathrm{YR} 7 / 3$ & $10 \mathrm{YR} 5 / 4$ & $\mathrm{mmg}$ & sl & abk & s.h & ss, ps & $\mathrm{ff}$ & es & aw \\
\hline $2 \mathrm{C} 3$ & $35-110$ & $10 \mathrm{YR} 7 / 3$ & $10 \mathrm{YR} 5 / 4$ & vfmg & $\mathrm{sl}$ & abk & $\mathrm{h}$ & ss, ps & - & es & - \\
\hline \multicolumn{12}{|c|}{ Profile No.12, (79.8 m a.s.l) } \\
\hline $\mathrm{C} 1$ & $0-20$ & $10 \mathrm{YR} 7 / 3$ & $10 \mathrm{YR} \mathrm{5/6}$ & $\mathrm{mmg}$ & sl & sbk & $\mathrm{s}$ & ss, ps & $\mathrm{mm}$ & es & as \\
\hline $\mathrm{C} 2$ & $20-70$ & $10 \mathrm{YR} 7 / 4$ & $10 \mathrm{YR} 5 / 6$ & amg & ls & $\mathrm{sg}$ & 1 & ss, po & $\mathrm{ff}$ & es & aw \\
\hline $2 \mathrm{C} 3$ & $70-130$ & $10 \mathrm{YR} 7 / 3$ & $10 \mathrm{YR} 5 / 4$ & amg & ls & $\mathrm{sg}$ & 1 & ss, po & - & es & - \\
\hline \multicolumn{12}{|c|}{ Profile No.13, (81.5 m a.s.l) } \\
\hline $\mathrm{C} 1$ & $0-20$ & $10 \mathrm{YR} 7 / 4$ & $10 \mathrm{YR} 5 / 6$ & $\mathrm{mmg}$ & $\mathrm{sl}$ & sbk & $\mathrm{s}$ & ss, ps & $\mathrm{mm}$ & es & as \\
\hline $\mathrm{C} 2$ & $20-50$ & $10 \mathrm{YR} 7 / 3$ & 10 YR 5/4 & $\mathrm{mmg}$ & ls & $\mathrm{sg}$ & 1 & ss, po & $\mathrm{ff}$ & es & aw \\
\hline $2 \mathrm{C} 3$ & $50-130$ & $10 \mathrm{YR} 7 / 3$ & 10 YR 5/4 & amg & ls & $\mathrm{sg}$ & 1 & ss, po & - & es & - \\
\hline
\end{tabular}




\section{Table 1: Cont}

\begin{tabular}{|c|c|c|c|c|c|c|c|c|c|c|c|}
\hline \multirow{2}{*}{ Horizon } & \multirow{2}{*}{$\begin{array}{l}\text { Depth } \\
\text { (cm) }\end{array}$} & \multicolumn{2}{|c|}{ Colour } & \multirow{2}{*}{$\begin{array}{c}\text { Coarse } \\
\text { fragments } \\
\text { Vol }(\%)^{1}\end{array}$} & \multirow{2}{*}{$\begin{array}{l}\text { Texture } \\
\text { USDA }^{2}\end{array}$} & \multirow{2}{*}{ Structure $^{3}$} & \multicolumn{2}{|c|}{ Consistence $^{4}$} & \multirow{2}{*}{ Roots $^{5}$} & \multirow{2}{*}{$\begin{array}{c}\text { Reaction } \\
\text { with } \mathrm{HCl}^{6}\end{array}$} & \multirow{2}{*}{ Boundary } \\
\hline & & Dry & Moist & & & & Dry & Wet & & & \\
\hline \multicolumn{12}{|c|}{ Profile No.14, (86.9 m a.s.l) } \\
\hline $\mathrm{C} 1$ & $0-15$ & $10 \mathrm{YR} 7 / 4$ & $10 \mathrm{YR} 5 / 6$ & $\mathrm{cmg}$ & ls & $\mathrm{m}$ & $\mathrm{s}$ & ss, po & $\mathrm{mm}$ & es & as \\
\hline $\mathrm{C} 2$ & $15-35$ & $10 \mathrm{YR} 7 / 4$ & $10 \mathrm{YR} 5 / 6$ & $\mathrm{cmg}$ & $\mathrm{s}$ & $\mathrm{sg}$ & 1 & so, po & $\mathrm{mm}$ & es & cs \\
\hline $2 \mathrm{C} 3$ & $35-110$ & $10 \mathrm{YR} 7 / 3$ & $10 \mathrm{YR} 5 / 4$ & $\mathrm{cmg}$ & $\mathrm{s}$ & sg & 1 & so, po & - & es & - \\
\hline \multicolumn{12}{|c|}{ Profile No.15, (84.0 m a.s.l) } \\
\hline $\mathrm{C} 1$ & $0-20$ & $10 \mathrm{YR} 7 / 6$ & $10 \mathrm{YR} 5 / 6$ & $\mathrm{cmg}$ & $\mathrm{s}$ & $\mathrm{m}$ & $\mathrm{s}$ & so, po & $\mathrm{mm}$ & es & as \\
\hline $2 \mathrm{C} 2$ & $20-50$ & $10 \mathrm{YR} 7 / 4$ & 10 YR 5/6 & $\mathrm{mmg}$ & $\mathrm{s}$ & sg & 1 & so, po & $\mathrm{ff}$ & es & aw \\
\hline $\mathrm{C} 3$ & $50-130$ & $10 \mathrm{YR} 7 / 3$ & $10 \mathrm{YR} 5 / 4$ & fmg & 1s & $\mathrm{m}$ & $\mathrm{s}$ & ss, po & - & es & - \\
\hline \multicolumn{12}{|c|}{ Profile No.16, (71.8 m a.s.l) } \\
\hline $\mathrm{C} 1$ & $0-40$ & $10 \mathrm{YR} 5 / 3$ & $10 \mathrm{YR} 3 / 4$ & $\mathrm{mmg}$ & sl & sbk & $\mathrm{s}$ & ss, ps & $\mathrm{mm}$ & es & as \\
\hline $\mathrm{C} 2$ & $40-65$ & $10 \mathrm{YR} 7 / 4$ & $10 \mathrm{YR} 5 / 6$ & $\mathrm{cmg}$ & ls & $\mathrm{sg}$ & 1 & ss, po & - & es & aw \\
\hline $\mathrm{C} 3$ & $65-130$ & $10 \mathrm{YR} 7 / 3$ & $10 \mathrm{YR} 5 / 4$ & $\mathrm{cmg}$ & $\mathrm{s}$ & $\mathrm{sg}$ & 1 & so, po & - & es & - \\
\hline
\end{tabular}

\section{Abbreviations:}

1: vfmg-very few medium gravel, fmg- few medium gravel, cmg-common medium gravel, mmg-many medium gravel, amg-abundant medium gravel

2: s-sand, l-loam, sl-sandy loam, ls-loamy sand, scl-sand clay loam, sil-silt loam

3: sg-single grain, m-massive, sbk-subangular blocky, abk-angular blocky

4: 1-loos, s-soft, sh-slightly hard, h-hard, vf- very hard, so-non sticky, po-non plastic, ss-slightly sticky, ps-slightly plastic, s-sticky, p- plastic

5: mm-many medium, mc-many coarse, ff-few fine

6: es-strongly effervescent, e-slightly effervescent, ev- violently effervescent

7: as-abrupt smooth, aw-abrupt wavy, cs-clear smooth, ai-abrupt irregular 
Table 2: Frequency distribution of heavy minerals of the studied soils

\begin{tabular}{|c|c|c|c|c|c|c|c|c|c|c|c|c|c|c|c|}
\hline \multirow[b]{2}{*}{ 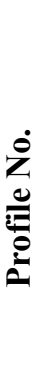 } & \multirow[b]{2}{*}{ ڤે } & \multirow[b]{2}{*}{ 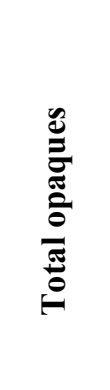 } & \multirow[b]{2}{*}{ 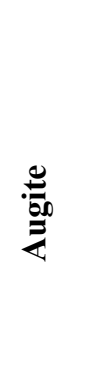 } & \multirow[b]{2}{*}{ 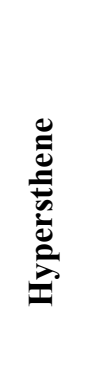 } & \multirow[b]{2}{*}{ 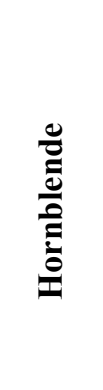 } & \multirow[b]{2}{*}{$\frac{\frac{0}{0}}{\frac{0}{0}}$} & \multicolumn{3}{|c|}{ Ubiquitous } & \multirow[b]{2}{*}{ 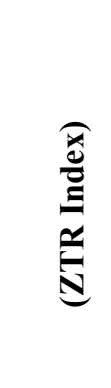 } & \multicolumn{2}{|c|}{$\begin{array}{c}\text { Parametamor- } \\
\text { phic }\end{array}$} & \multirow[b]{2}{*}{ हేّ } & \multirow[b]{2}{*}{ 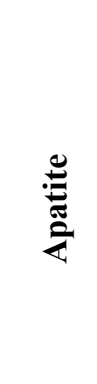 } & \multirow[b]{2}{*}{$\stackrel{0}{0}$} \\
\hline & & & & & & & 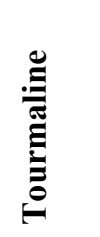 & $\underset{0}{\stackrel{0}{*}}$ & $\stackrel{\mathscr{D}}{\stackrel{\Xi}{\Xi}}$ & & $\underset{\Xi}{\overparen{E}}$ & 节 & & & \\
\hline \multirow{3}{*}{1} & $0-15$ & 14.54 & 2.08 & 1.04 & 1.39 & 4.51 & 27.43 & 43.75 & 4.17 & 75.35 & 16.67 & 0.00 & 16.67 & 0.00 & 3.47 \\
\hline & $15-45$ & 41.06 & 3.45 & 2.07 & 7.59 & 13.10 & 18.62 & 51.03 & 2.76 & 72.41 & 12.41 & 0.00 & 12.41 & 0.00 & 2.07 \\
\hline & $45-75$ & 32.38 & 10.91 & 0.00 & 12.73 & 23.64 & 13.33 & 34.55 & 3.03 & 50.91 & 23.03 & 0.00 & 23.03 & 0.00 & 2.42 \\
\hline \multirow{3}{*}{2} & $0-10$ & 51.63 & 7.98 & 0.00 & 5.52 & 13.50 & 23.93 & 25.77 & 2.45 & 52.15 & 32.52 & 0.00 & 32.52 & 0.00 & 1.84 \\
\hline & $10-20$ & 64.37 & 3.23 & 0.00 & 7.26 & 10.48 & 15.32 & 50.00 & 0.81 & 66.13 & 13.71 & 0.00 & 13.71 & 0.00 & 9.68 \\
\hline & $20-40$ & 60.00 & 3.19 & 3.72 & 10.11 & 17.02 & 44.68 & 22.87 & 6.91 & 74.47 & 5.85 & 0.00 & 5.85 & 0.00 & 2.66 \\
\hline \multirow{3}{*}{3} & $0-15$ & 65.20 & 6.34 & 4.93 & 4.23 & 15.49 & 33.10 & 26.06 & 13.38 & 72.54 & 9.86 & 0.00 & 9.86 & 0.00 & 2.11 \\
\hline & $15-35$ & 54.48 & 7.58 & 1.52 & 21.21 & 30.30 & 18.18 & 33.33 & 4.55 & 56.06 & 7.58 & 0.00 & 7.58 & 0.00 & 6.06 \\
\hline & $35-110$ & 57.18 & 13.17 & 5.39 & 2.40 & 20.96 & 14.37 & 31.14 & 5.39 & 50.90 & 4.79 & 0.00 & 4.79 & 15.57 & 7.78 \\
\hline \multirow{3}{*}{4} & $0-15$ & 59.43 & 4.82 & 2.63 & 5.26 & 12.72 & 29.39 & 37.28 & 7.89 & 74.56 & 3.51 & 0.00 & 3.51 & 3.07 & 6.14 \\
\hline & $15-55$ & 56.26 & 4.17 & 1.56 & 4.17 & 9.90 & 12.50 & 34.38 & 9.90 & 56.77 & 27.08 & 0.00 & 27.08 & 0.00 & 6.25 \\
\hline & $55-80$ & 51.63 & 4.49 & 0.00 & 9.55 & 14.04 & 15.17 & 34.83 & 7.30 & 57.30 & 20.79 & 0.00 & 20.79 & 0.00 & 7.87 \\
\hline
\end{tabular}


Table 2: Cont.

\begin{tabular}{|c|c|c|c|c|c|c|c|c|c|c|c|c|c|c|c|c|}
\hline \multirow[b]{2}{*}{ 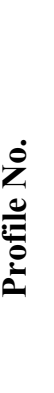 } & \multirow[b]{2}{*}{ 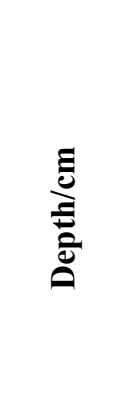 } & \multirow[b]{2}{*}{ 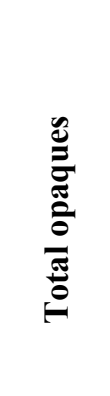 } & \multirow[b]{2}{*}{ 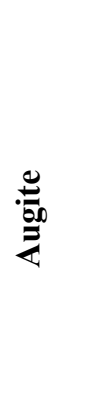 } & \multirow[b]{2}{*}{ 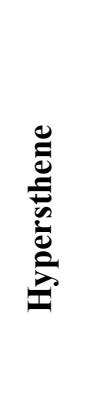 } & \multirow[b]{2}{*}{ 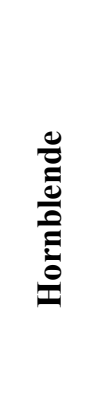 } & \multirow[b]{2}{*}{$\begin{array}{l}\frac{0}{0} \\
\frac{0}{0} \\
\frac{0}{2}\end{array}$} & \multicolumn{3}{|c|}{ Ubiquitous } & \multirow[b]{2}{*}{ 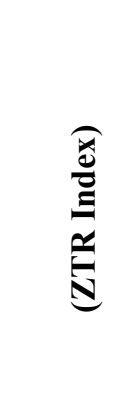 } & \multicolumn{2}{|c|}{$\begin{array}{c}\text { Parametamor- } \\
\text { phic }\end{array}$} & \multirow[b]{2}{*}{$\stackrel{\tilde{T}}{\theta}$} & \multirow[b]{2}{*}{ 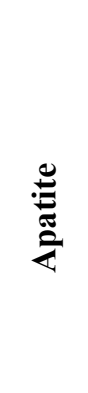 } & \multirow[b]{2}{*}{ 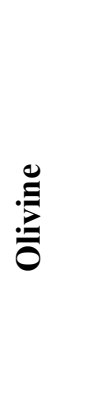 } & \multirow[b]{2}{*}{$\frac{0}{0}$} \\
\hline & & & & & & & 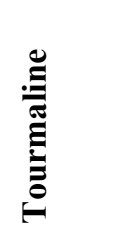 & $\stackrel{\Xi}{\stackrel{0}{*}}$ & 䒿 & & 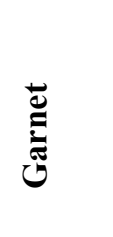 & : & & & & \\
\hline \multirow{2}{*}{5} & $0-10$ & 59.49 & 3.13 & 1.56 & 8.33 & 13.02 & 22.40 & 25.00 & 12.50 & 59.90 & 19.79 & 0.00 & 19.79 & 0.00 & 0.00 & 7.29 \\
\hline & $10-30$ & 59.45 & 4.30 & 1.79 & 8.96 & 15.05 & 20.07 & 31.18 & 10.04 & 61.29 & 17.56 & 0.00 & 17.56 & 0.00 & 0.00 & 6.09 \\
\hline \multirow{2}{*}{6} & $15-95$ & 58.04 & 1.40 & 0.93 & 8.88 & 11.21 & 22.43 & 31.31 & 3.27 & 57.01 & 26.17 & 0.00 & 26.17 & 0.00 & 0.00 & 5.61 \\
\hline & $95-115$ & 54.50 & 1.16 & 0.00 & 4.65 & 5.81 & 27.33 & 27.33 & 0.58 & 55.23 & 36.63 & 0.00 & 36.63 & 0.00 & 0.00 & 2.33 \\
\hline \multirow{2}{*}{7} & $30-85$ & 54.86 & 1.74 & 0.29 & 12.21 & 14.24 & 20.64 & 31.10 & 4.65 & 56.40 & 25.87 & 0.00 & 25.87 & 0.00 & 0.00 & 3.49 \\
\hline & $85-140$ & 55.05 & 2.04 & 0.00 & 7.40 & 9.44 & 18.37 & 37.50 & 5.36 & 61.22 & 23.72 & 2.30 & 26.02 & 0.00 & 0.00 & 3.32 \\
\hline \multirow{3}{*}{8} & $0-15$ & 44.47 & 2.31 & 0.00 & 7.41 & 9.72 & 22.69 & 33.33 & 1.39 & 57.41 & 25.93 & 1.85 & 27.78 & 0.00 & 0.00 & 5.09 \\
\hline & $15-55$ & 44.14 & 2.95 & 0.00 & 5.90 & 8.85 & 18.69 & 41.31 & 4.59 & 64.59 & 17.05 & 7.21 & 24.26 & 0.00 & 0.00 & 2.30 \\
\hline & $55-110$ & 44.78 & 2.18 & 0.00 & 10.18 & 12.36 & 34.91 & 24.73 & 2.18 & 61.82 & 18.55 & 2.91 & 21.45 & 0.00 & 0.00 & 4.36 \\
\hline \multirow{3}{*}{9} & $0-15$ & 53.35 & 3.80 & 0.84 & 5.49 & 10.13 & 25.53 & 27.85 & 5.49 & 58.86 & 23.00 & 3.16 & 26.16 & 0.00 & 0.00 & 4.85 \\
\hline & $15-35$ & 57.75 & 1.27 & 0.00 & 2.53 & 3.80 & 17.30 & 53.16 & 2.53 & 73.00 & 20.25 & 0.84 & 21.10 & 0.00 & 0.00 & 2.11 \\
\hline & $35-70$ & 65.63 & 2.58 & 0.65 & 7.74 & 10.97 & 25.16 & 30.32 & 5.16 & 60.65 & 22.58 & 0.00 & 22.58 & 1.94 & 0.00 & 3.87 \\
\hline
\end{tabular}


Table 2: Cont.

\begin{tabular}{|c|c|c|c|c|c|c|c|c|c|c|c|c|c|c|c|c|}
\hline \multirow[b]{2}{*}{ 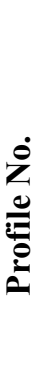 } & \multirow[b]{2}{*}{ 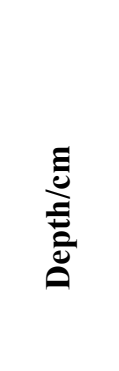 } & \multirow[b]{2}{*}{ 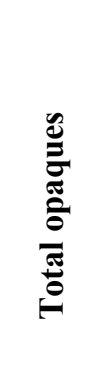 } & \multirow[b]{2}{*}{ 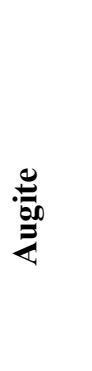 } & \multirow[b]{2}{*}{ 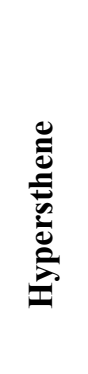 } & \multirow[b]{2}{*}{ 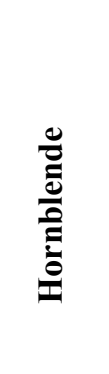 } & \multirow[b]{2}{*}{$\begin{array}{l}\frac{n}{0} \\
\frac{0}{0} \\
\frac{0}{0} \\
0\end{array}$} & \multicolumn{3}{|c|}{ Ubiquitous } & \multirow[b]{2}{*}{ 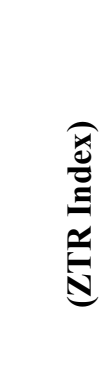 } & \multicolumn{2}{|c|}{$\begin{array}{c}\text { Parametamor- } \\
\text { phic }\end{array}$} & \multirow[b]{2}{*}{$\stackrel{\vec{\pi}}{\theta}$} & \multirow[b]{2}{*}{ 䒿 } & \multirow[b]{2}{*}{ :. } & \multirow[b]{2}{*}{ 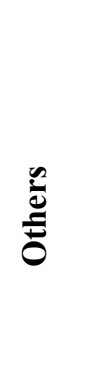 } \\
\hline & & & & & & & 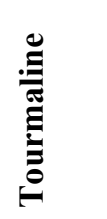 & $\stackrel{\Xi}{\stackrel{\Xi}{*}}$ & $\stackrel{0}{\stackrel{0}{E}}$ & & 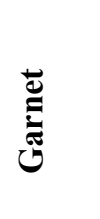 & 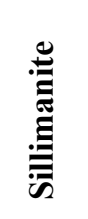 & & & & \\
\hline \multirow{3}{*}{10} & $0-20$ & 58.31 & 4.37 & 3.83 & 7.10 & 15.30 & 18.58 & 34.97 & 4.92 & 58.47 & 22.95 & 0.00 & 22.95 & 0.00 & 0.00 & 3.28 \\
\hline & $20-60$ & 55.63 & 3.76 & 0.00 & 12.21 & 15.96 & 22.54 & 26.76 & 12.21 & 61.50 & 17.37 & 0.00 & 17.37 & 0.00 & 0.00 & 5.16 \\
\hline & $60-120$ & 60.49 & 4.31 & 0.00 & 6.22 & 10.53 & 25.84 & 21.53 & 10.05 & 57.42 & 24.88 & 0.00 & 24.88 & 0.00 & 0.00 & 7.18 \\
\hline \multirow{2}{*}{11} & $0-15$ & 56.06 & 8.13 & 0.00 & 3.89 & 12.01 & 22.61 & 39.58 & 4.59 & 66.78 & 14.84 & 0.00 & 14.84 & 0.00 & 0.00 & 6.36 \\
\hline & $15-35$ & 64.93 & 4.00 & 0.57 & 9.14 & 13.71 & 20.57 & 36.57 & 6.29 & 63.43 & 18.29 & 0.00 & 18.29 & 0.00 & 0.00 & 4.57 \\
\hline \multirow{2}{*}{12} & $0-20$ & 57.80 & 2.99 & 0.00 & 4.10 & 7.09 & 25.37 & 45.15 & 3.36 & 73.88 & 16.42 & 0.00 & 16.42 & 0.00 & 0.00 & 2.61 \\
\hline & $20-70$ & 47.54 & 1.56 & 1.04 & 2.60 & 5.21 & 21.35 & 35.94 & 6.77 & 64.06 & 29.69 & 0.00 & 29.69 & 0.00 & 0.00 & 1.04 \\
\hline \multirow{3}{*}{13} & $0-20$ & 60.26 & 1.31 & 0.65 & 8.50 & 10.46 & 22.22 & 37.91 & 5.88 & 66.01 & 18.95 & 0.00 & 18.95 & 1.31 & 0.00 & 3.27 \\
\hline & $20-50$ & 62.28 & 1.68 & 0.00 & 7.72 & 9.40 & 39.60 & 28.86 & 5.70 & 74.16 & 11.41 & 0.00 & 11.41 & 0.00 & 0.00 & 5.03 \\
\hline & $50-130$ & 65.63 & 2.73 & 0.00 & 8.18 & 10.91 & 17.27 & 39.09 & 3.64 & 60.00 & 19.09 & 0.00 & 19.09 & 0.00 & 0.00 & 10.00 \\
\hline \multirow{2}{*}{14} & $0-15$ & 89.68 & 0.75 & 0.00 & 2.24 & 2.99 & 16.79 & 59.70 & 5.60 & 82.09 & 11.94 & 0.00 & 11.94 & 0.00 & 0.00 & 2.99 \\
\hline & $15-35$ & 57.96 & 2.55 & 0.00 & 11.06 & 13.62 & 32.34 & 24.26 & 5.53 & 62.13 & 19.15 & 0.00 & 19.15 & 0.00 & 0.00 & 5.11 \\
\hline
\end{tabular}


Table 2: Cont.

\begin{tabular}{|c|c|c|c|c|c|c|c|c|c|c|c|c|c|c|c|c|}
\hline \multirow{2}{*}{ 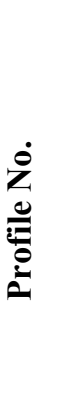 } & \multirow[b]{2}{*}{ 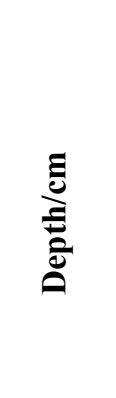 } & \multirow{2}{*}{ 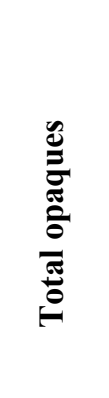 } & \multirow[b]{2}{*}{ 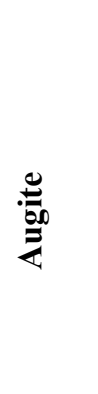 } & \multirow{2}{*}{ 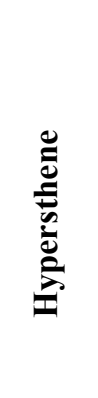 } & \multirow{2}{*}{ 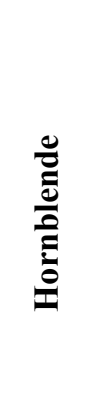 } & \multirow[b]{2}{*}{$\begin{array}{l}\frac{0}{0} \\
\frac{0}{0} \\
\frac{0}{2}\end{array}$} & \multicolumn{3}{|c|}{ Ubiquitous } & \multirow{2}{*}{ 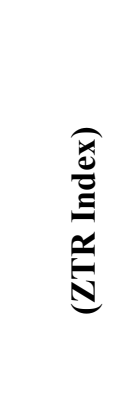 } & \multicolumn{2}{|c|}{$\begin{array}{l}\text { Parametamor- } \\
\text { phic }\end{array}$} & \multirow[b]{2}{*}{ 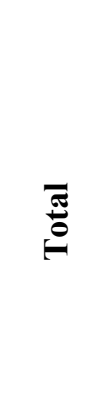 } & \multirow[b]{2}{*}{ 䓂 } & \multirow[b]{2}{*}{ 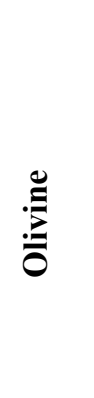 } & \multirow[b]{2}{*}{$\frac{n}{\tilde{\Xi}}$} \\
\hline & & & & & & & & : & 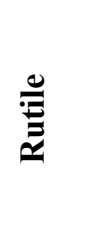 & & 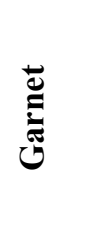 & 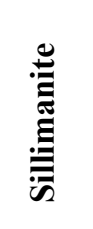 & & & & \\
\hline \multirow{2}{*}{15} & $0-20$ & 46.46 & 2.94 & 0.00 & 8.82 & 11.76 & 27.94 & 21.57 & 12.75 & 62.25 & 15.69 & 0.00 & 15.69 & 0.00 & 0.00 & 10.29 \\
\hline & $20-50$ & 46.00 & 3.10 & 0.69 & 11.72 & 15.52 & 29.31 & 29.66 & 4.14 & 63.10 & 12.41 & 0.00 & 12.41 & 0.00 & 0.00 & 8.97 \\
\hline \multirow{2}{*}{16} & $0-40$ & 50.51 & 2.07 & 0.00 & 5.52 & 7.59 & 17.93 & 48.97 & 6.21 & 73.10 & 12.41 & 0.00 & 12.41 & 0.00 & 0.00 & 6.90 \\
\hline & $40-65$ & 63.00 & 2.64 & 0.00 & 7.59 & 10.23 & 20.46 & 54.46 & 3.63 & 78.55 & 4.29 & 0.00 & 4.29 & 0.00 & 0.00 & 6.93 \\
\hline
\end{tabular}


showed increasing with depth at the ground elevations between 71.8 to $86.9 \mathrm{~m}$ a.s.l and discontinuity allocation between 59.4 to $81.5 \mathrm{~m}$ a.s.1. Frequency distribution of Augite correlated positively with Hypersthene $\left(\mathrm{r}=0.443^{* *}\right)$ and Apatite $\left(\mathrm{r}=0.564^{* *}\right)$ while correlated negatively with Tourmaline $(\mathrm{r}=-$ $0.250)$.

Tourmaline is present in all studied soil samples where its percentages ranged from $44.68 \%$ at subsurface soils of (profile 2; $61.9 \mathrm{~m}$ a.s.1) to $12.50 \%$ at $2 \mathrm{C} 2$ horizon of (soil profile $4 ; 76.4 \mathrm{~m}$ a.s.l). Statistically, it was noticed that there is a moderate negative correlation between Tourmaline and Zircon $\left(\mathrm{r}=-0.518^{* *}\right)$.

Zircon is commonly found in considerable amounts within the examined samples where it showed a minimum quantity of $21.53 \%$ at the subsurface soils of profile 10 which has a ground elevation of 71.6 $m$ a.s.l. While, the higher frequency content of Zircon (59.70\%) was observed at the top soils of profile 14 that formed on earth elevation of $86.9 \mathrm{~m}$ a.s.l. A negative correlation relationship was significantly observed between Zircon and Rutile $\left(\mathrm{r}=-0.409^{* *}\right)$.

Rutile is mostly detected in horizons of the studied profiles with quantities smaller than both of Zircon and Tourmaline. However, it was observed in higher contents at surface soils of profile (3) at ground elevation of $59.4 \mathrm{~m}$ a.s.l while its lower frequency was distinguished at subsurface horizon (95$115 \mathrm{~cm})$ of profile 6 which is representing to the most high land elevation $(111.1 \mathrm{~m}$ a.s.l) within the studied area.

Garnet fluctuated between $3.51 \%$ in profile 4 ( $76.4 \mathrm{~m}$ a.s.1) and $36.63 \%$ in $2 \mathrm{C} 3$ horizon of profile 6 (111.1 m a.s.l). Also, it is found in high contents within soil profiles $(2,12,4,6,7,8$, and 10) which are exemplifying elevations of $(61.9-111.1 \mathrm{~m}$ a.s.l) on the topo-sequence model of the research site. A significant negative correlation was predestine between Garnet and Apatite $\left(r=-0.325^{*}\right)$.

Sillimanite is predominately distinguished in inconsiderable amounts or not observed. Where, its higher content was $7.21 \%$ at subsurface soils of profile $8(67.5 \mathrm{~m}$ a.s.l). According to Garnet and Sillimanite frequencies, parametamorphic minerals varied between 3.51 to $36.63 \%$ within the research area and showed decreasing trend with depth in between ground elevations 59.4 to $84 \mathrm{~m}$ a.s.l. On the other hand, they displayed an irregular vertical distribution in between 63.4 to $81.5 \mathrm{~m}$ a.s.l.

Apatite is commonly imperceptible in most of the estimated samples; however it was only noticed in subsurface soils of profiles (3; $59.4 \mathrm{~m}$ a.s.1.) \& $(9 ; 71.8 \mathrm{~m}$ a.s.1.) and in surface soils of profiles (4; $76.4 \mathrm{~m}$ a.s.1.) \& (13; $81.5 \mathrm{~m}$ a.s.1.). Where, its content not exceeded $16 \%$. In addition to the previously described minerals, other minerals such as Olivine, Monazite, and Biotite were detected in minute amounts.

\subsection{Soil genesis and maturity evaluation}

The results revealed that ZTR index values were commonly less than $75 \%$ (Table 2), indicating that the studied soils are less impacted by weathering, immature, weakly developed, recently formed and have low effect of soil forming processes.

\subsection{Parent material uniformity appraisal}

Investigation of heavy minerals is counted as standard for evaluating genesis and homogeneity of soil parent material. Haseman and Marshall (1945), Hammad (1968), El Kady (2008), El Kady and Yossif (2017) and others used the ratio between zircon/tourmaline (Z/T), zircon/rutile (Z/R), and zircon/ rutile + tourmaline $(\mathrm{Z} / \mathrm{R}+\mathrm{T})$ to demonstrate uniformity or discontinuity of parent material. Based on them, soils of the studied area are heterogeneous owing to multi-source and their depositional regimes (Fig.4). Theses lithological discontinuities were matching with soil morphological observations.

Cherian et al. (2004) and Kasper-Zubillaga et al. (2005) recorded some methods such as microscopic analyses to evaluate mode of transportation and consequently genesis of the sediments through the shape and surface features of the grains. By applying these methods on heavy minerals samples in the study area (Plate 1) shows that they are mostly sub-angular to sub-rounded with some samples rounded to well rounded. These means that there are at least two sources of sediments that transported by wind from nearby source and re-worked ones (rounded to well-rounded) by water from 
source not far away. They supported by the presence of polishing on the surface of sub-angular to angular samples and pitted surface of rounded to well-rounded ones.

Parent material heterogeneity of the study area encourage to use particular system for land use such as (1) using row crop with occasional close seeded soil conserving crop, (2) returning crop residue to the soil, (3) employing of practice conservation tillage like utilizing crop residue as a mulch, and (4) removing undesirable brush and trees more than $5 \mathrm{~cm}$ in diameter at $150 \mathrm{~cm}$ above ground.

Table 3: Pearson correlation coefficients between the estimated heavy minerals in the studied soil profiles

\begin{tabular}{|c|c|c|c|c|c|c|c|c|c|c|c|c|}
\hline \multicolumn{2}{|c|}{ Mineral } & 1 & 2 & 3 & 4 & 5 & 6 & 7 & 8 & 9 & 10 & 11 \\
\hline 1 & Total Opaques & 1 & & & & & & & & & & \\
\hline 2 & Augite & -0.137 & 1 & & & & & & & & & \\
\hline 3 & Hypersthene & 0.083 & $0.443^{* *}$ & 1 & & & & & & & & \\
\hline 4 & Horneblende & -0.057 & 0.106 & -0.130 & 1 & & & & & & & \\
\hline 5 & Tourmaline & -0.019 & -0.250 & 0.132 & 0.063 & 1 & & & & & & \\
\hline 6 & Zircon & 0.120 & -0.210 & -0.20 & $-0.348^{*}$ & $-0.518^{* *}$ & 1 & & & & & \\
\hline 7 & Rutile & 0.191 & 0.081 & $0.319^{*}$ & 0.024 & 0.142 & $-0.409^{* *}$ & 1 & & & & \\
\hline 8 & Garnet & -0.180 & -0.220 & $-0.398^{*}$ & -0.110 & -0.190 & -0.280 & -0.180 & 1 & & & \\
\hline 9 & Sillimanite & -0.220 & -0.130 & -0.180 & -0.070 & -0.020 & 0.018 & -0.180 & 0.095 & 1 & & \\
\hline 10 & Apatite & 0.067 & $0.564^{* *}$ & $0.542^{* *}$ & -0.230 & -0.170 & -0.060 & 0.004 & $-0.325^{*}$ & -0.080 & 1 & \\
\hline 11 & Others & 0.128 & 0.113 & -0.090 & 0.223 & -0.140 & -0.110 & 0.222 & -0.300 & -0.210 & 0.180 & 1 \\
\hline
\end{tabular}

**Correlation is significant at the 0.01 level $(\mathrm{p}<0.01)$

*Correlation is significant at the 0.05 level $(\mathrm{p}<0.05)$

Table 4: Ratios of the resistant minerals in the separated sand at the studied profiles

\begin{tabular}{|c|c|c|c|c|c|c|c|c|c|}
\hline Profile No. & Depth/cm & $\mathbf{Z} / \mathbf{R}$ & $\mathbf{Z} / \mathbf{T}$ & $\mathbf{Z} / \mathbf{R}+\mathbf{T}$ & Profile No. & Depth/cm & $\mathbf{Z} / \mathbf{R}$ & $\mathbf{Z} / \mathbf{T}$ & $\mathrm{Z} / \mathrm{R}+\mathrm{T}$ \\
\hline \multirow{3}{*}{1} & $0-15$ & 10.50 & 1.59 & 1.38 & \multirow{3}{*}{9} & $0-15$ & 5.08 & 1.09 & 0.90 \\
\hline & $15-45$ & 18.50 & 2.74 & 2.39 & & $15-35$ & 21.00 & 3.07 & 2.68 \\
\hline & $45-75$ & 11.40 & 2.59 & 2.11 & & $35-70$ & 5.88 & 1.21 & 1.00 \\
\hline \multirow{3}{*}{2} & $0-10$ & 10.50 & 1.08 & 0.98 & \multirow{3}{*}{10} & $0-20$ & 7.11 & 1.88 & 1.49 \\
\hline & $10-20$ & 62.00 & 3.26 & 3.10 & & $20-60$ & 2.19 & 1.19 & 0.77 \\
\hline & $20-40$ & 3.31 & 0.51 & 0.44 & & $60-120$ & 2.14 & 0.83 & 0.60 \\
\hline \multirow{3}{*}{3} & $0-15$ & 1.95 & 0.79 & 0.56 & \multirow{2}{*}{11} & $0-15$ & 8.62 & 1.75 & 1.45 \\
\hline & $15-35$ & 7.33 & 1.83 & 1.47 & & $15-35$ & 5.82 & 1.78 & 1.36 \\
\hline & $35-110$ & 5.78 & 2.17 & 1.58 & \multirow{2}{*}{12} & $0-20$ & 13.44 & 1.78 & 1.57 \\
\hline \multirow{3}{*}{4} & $0-15$ & 4.72 & 1.27 & 1.00 & & $20-70$ & 5.31 & 1.68 & 1.28 \\
\hline & $15-55$ & 3.47 & 2.75 & 1.53 & \multirow{3}{*}{13} & $0-20$ & 6.44 & 1.71 & 1.35 \\
\hline & $55-80$ & 4.77 & 2.30 & 1.55 & & $20-50$ & 5.06 & 0.73 & 0.64 \\
\hline \multirow[b]{2}{*}{5} & $0-10$ & 2.00 & 1.12 & 0.72 & & $50-130$ & 10.75 & 2.26 & 1.87 \\
\hline & $10-30$ & 3.11 & 1.55 & 1.04 & \multirow{2}{*}{14} & $0-15$ & 10.67 & 3.56 & 2.67 \\
\hline \multirow[b]{2}{*}{6} & $15-95$ & 9.57 & 1.40 & 1.22 & & $15-35$ & 4.38 & 0.75 & 0.64 \\
\hline & $95-115$ & 47.00 & 1.00 & 0.98 & \multirow{2}{*}{15} & $0-20$ & 1.69 & 0.77 & 0.53 \\
\hline \multirow[b]{2}{*}{7} & $30-85$ & 6.69 & 1.51 & 1.23 & & $20-50$ & 7.17 & 1.01 & 0.89 \\
\hline & $85-140$ & 7.00 & 2.04 & 1.58 & \multirow{4}{*}{16} & $0-40$ & 7.89 & 2.73 & 2.03 \\
\hline \multirow{3}{*}{8} & $0-15$ & 24.00 & 1.47 & 1.38 & & & & & \\
\hline & $15-55$ & 9.00 & 2.21 & 1.77 & & $40-65$ & 15.00 & 2.66 & 2.26 \\
\hline & $55-110$ & 11.33 & 0.71 & 0.67 & & & & & \\
\hline
\end{tabular}




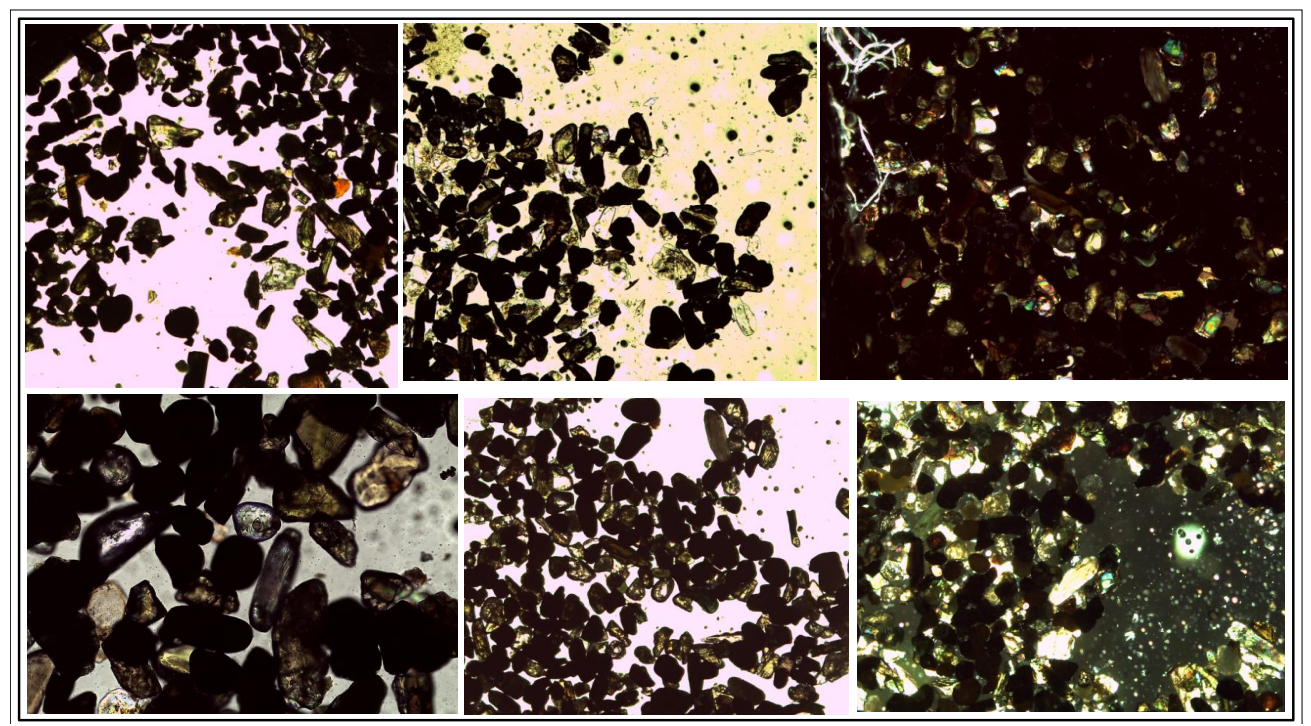

Plate 1: Some microscopic micrographs of the heavy minerals in the soils of the study area

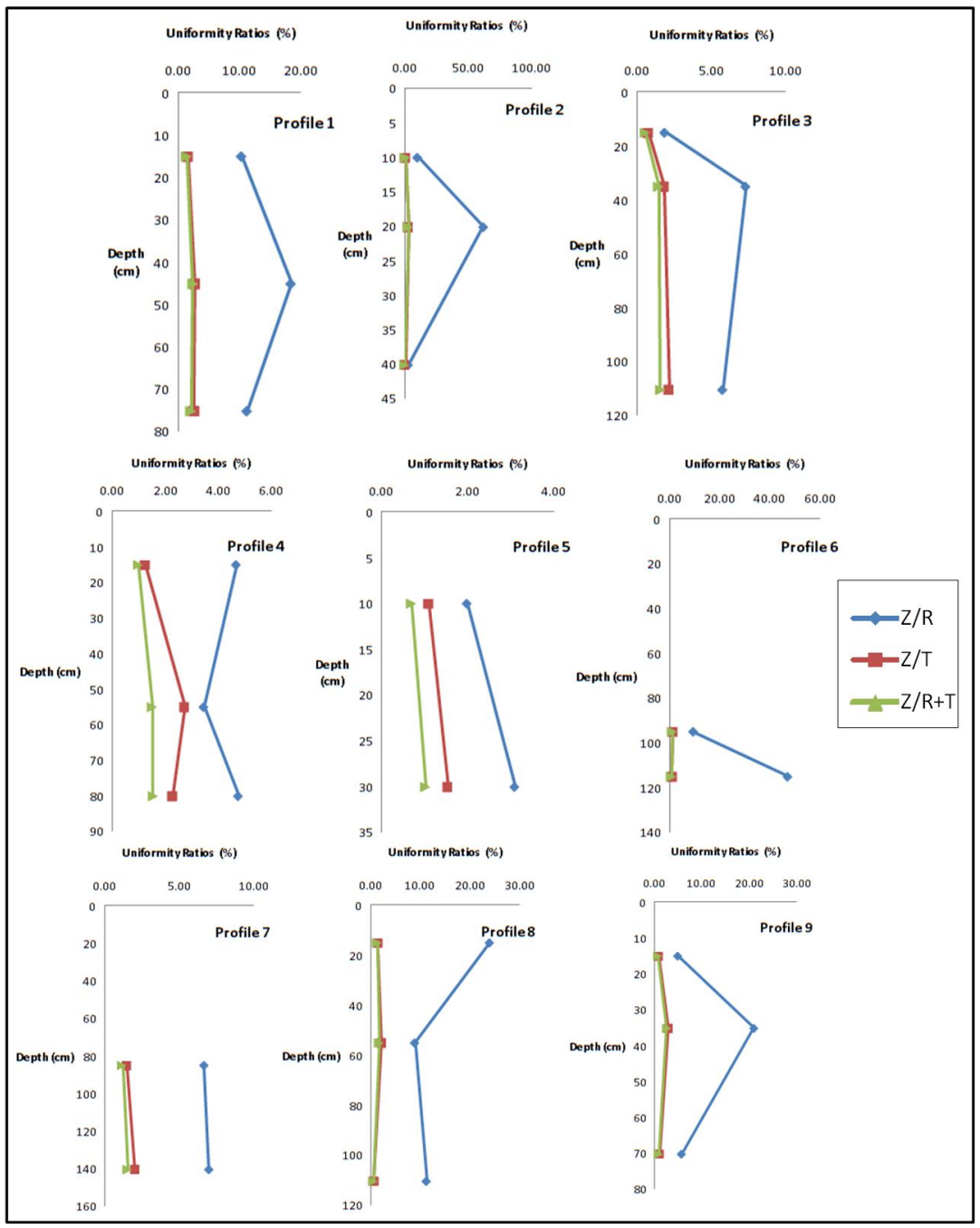




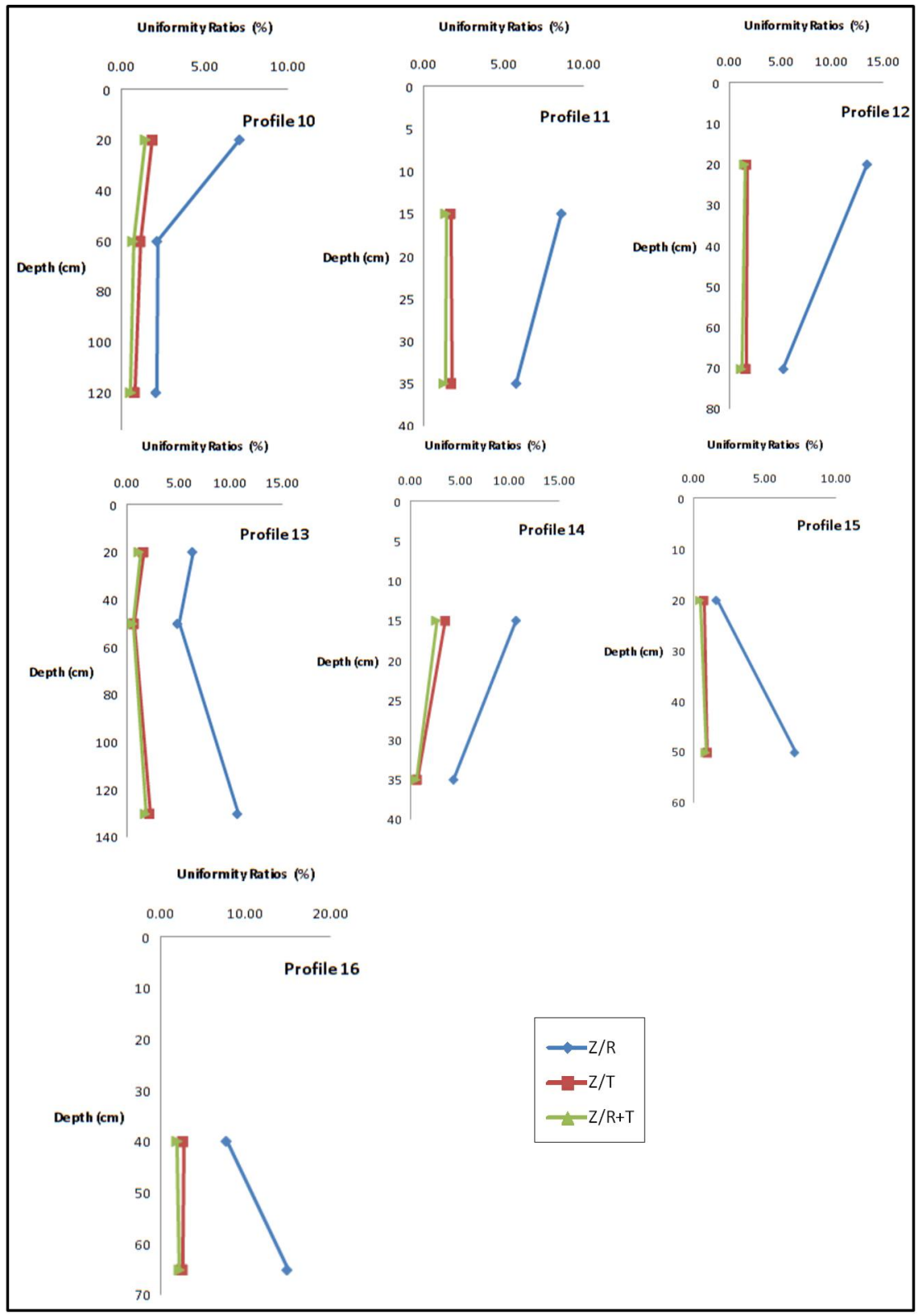

Fig. 4: Depth-wise patterns for uniformity ratios of the studied soils

\section{Conclusion}

The main goal of the paper is appraising of soil genesis and parent material uniformity on basis of a topo-sequence transect within some soils west Manfalut, Assuit-Egypt for precision agriculture. From the research outputs, it is possible to conclude that the investigated soils are recently formed and have low impact of soil forming processes. Furthermore, soil parent materials were diversified nature and apparently formed of heterogeneous depositional regimes. The findings pointed that there are at least two sources of sediments that transported by wind from nearby source and re-worked ones (rounded to well-rounded) by water from source not far away. Initially, these soils require moderate to special conservation practices that have to apply in terms of vegetative and possible mechanical treatments. Further study of landuse assessment for agriculture within the research area would be of interest. 


\section{References}

Abou El-Anwar, E.A., H.S. Mekky, S.A. Salman, A.A. Elnazer, W. Abdel Wahab, and A.S. Asmoay, 2019. Mineralogical and petrographical studies of agricultural soil, Assiut Governorate, Egypt. Bull. Natl. Res. Cent. 43, 30.

Barshad, I., 1965. Chemistry of soil development, F.E. Bear (Ed.), Chemistry of the Soil (2 ${ }^{\text {nd }}$ edn.), Reinhold Publ. Co., New York, 1-70.

Brewer, R., 1964. Fabric and mineral analysis of soils. John Wiley and Sons, Inc. New York, London. Brewer, R., 1976. Fabric and Mineral Analysis of Soils, Robert E. Krieger Publishing Co., New York.

Chapman, S.L., and M.E. Horn, 1968. Parent material uniformity and origin of silty soils in northwest Arkansas based on zirconium titanium contents. Soil Science Society of America Proceedings, 32: $265-271$.

Cherian, A.N.C. and V. Rajamanickam, 2004. Light minerals of beach sediments from Southern Tamilnadu, south east coast of India. Oceanologia, 46: 233-252.

El Kady, M.M and T.M. Yossif, 2017. Genesis and Parent Material Uniformity Appraisal of some Adjacent Soils to Bani Mazar-El Boiety Road, El Minia, Egypt. Alexandria Science Exchange Journal, 38 (3): 397-411

EL Kady, M.M., 2008. Soils Distribution and Development of El Alamein - Wadi El Natrun Area, Egypt. M.Sc. Thesis, Fac. Agric., Al-Azhar University, Cairo.

Geological survey of Egypt, 1981. Geological Map of Egypt, Scale 1:2,000,000 -Egyptian Geological Survey and Mining Authority; Cairo.

Gile, L.H., J.W. Hawley, and R.B. Grossman, 1981. Soils and geomor-phology in the Basin and Range area of Southern New Mexico: Guide book to the Desert Project. New Mexico Bureau of Mines \&Mineral Resources. Univ. of New Mexico, Socorro, 222 pages.

Hammad, M.A., 1968. Genesis of the soils of the Western Mediterranean Coast of U.A.R., Ph.D. Thesis, Fac. Agric., Ain Shams Univ., U.A.R.

Haseman, J.F. and C.E. Marshall, 1945. The use of heavy minerals in studies of the origin and development of soils. Missouri Agr. Exp. Sta. Res. Bull., 387.

Hubert, J.F., 1962. A Zircon-Tourmaline-Rutile Maturity Index and Independence of Composition of Heavy Mineral Assemblages with Gross Composition and Texture of Sandstone. Journal of Sedimentary Petrology, 32: 440-450.

Jay, A.S., 2015. Forensic chemistry: fundamental and applications. Foren. Sc., Mi., ISBN: 978-1-11889772-0, Wiley-Blackwell, 542.

Kasper-Zubillaga, J.J., W.W. Dickinson, A. Carranza-Edwards, and Y. Hornelas-Orozco, 2005. Petrography of quartz grains in beach and dune sands of Northland, North Island, New Zealand. New Zealand Journal of Geology and Geophysics, 48, 649-660.

Mahaney, W.C., 2002. Atlas of Sand Grain Surface Textures and Applications, Oxford University Press, Science, 237.

Mallik, T.K., 2018. A guide to rapid identification of heavy minerals and highlights of heavy mineral distribution pattern along Indian coasts. MOJ Eco Environ Sci. 3(4): 260-263.

Norton, L.D., and G.F. Hall, 1984. Differentiation of lithologically similar soil parent materials. SSSAJ, 49(2): 409-414.

Omer, A.A., 1996. Geological, mineralogical and geochemical studies on the Neogene and Quaternary Nile basin deposits, Qena-Assiut stretch, Egypt. PhD thesis, Geol Dept Fac Sci Sohag, South Valley Univ., 320.

Osman, H.Z., 1980. Geological studies on the area to the northwest of Assiut M.Sc. thesis, Fac. Sci., Assiut Univ., Assiut, Egypt, 276.

Raad, A.T., R. Prozt, 1971. A new method for the identification of sediment stratification in soils of the Blue Ridge Springs Basin, Ontario. Geoderma 6: 23-41.

Reid, H.M., 2013. Introduction to Statistics: Fundamental Concepts and Procedures of Data Analysis. SAGE Publications, USA, 632.

Said, R., 1962. The geology of Egypt. Elsevier Pub. Comp, Amesterdam, New York, 377.

Said, R., 1981. The geological evolution of the River Nile. Springer Verlag, New York, Heidelbeirg, Berlin, p 151. 
Schaetzl, R.J., 1998. Lithologic discontinuities in some soils on drumlins: theory, detection, and application. Soil Science, 163: 570-590.

Soil Survey Staff, 2009. Soil Survey Field and Laboratory Methods Manual. Soil Survey Investigations Report No. 51, Ver. 1.0. P. 1:405, Ed. R. Burt. U.S. Dep. of Agri., Nat. Res. Cons. Ser., Lincoln, Nebraska.

Taboada, T., A.M. Cortizas, C. Garcia, and E.G. Rodeja, 2006. Particle-size fractionation of titanium and zirconium during weathering and pedogenesis of granitic rocks in NW Spain. Geoderma, 131: 218-236.

USDA, 2014. In: "Keys to Soil Taxonomy". Soil Surv. Staff. Washingt, USA.

Wilson, S.G., J. Lambert, M. Nanzyo, and R.A. Dahlgren, 2017. Soil genesis and mineralogy across a volcanic lithosequence. Geoderma, 285: 301-312. 